Memorias del VII Encuentro Nacional de Experiencias en la Enseñanza de la Biología y la Educación Ambiental y II Congreso Nacional de Investigación en la Enseñanza de la Biología

\title{
APROXIMACIÓN A LAS CONCEPCIONES ACERCA DE ESPECIE DE ESTUDIANTES DE NOVENO GRADO DE UNA INSTITUCIÓN EDUCATIVA OFICIAL DE LA CIUDAD DE NEIVA
}

\section{APPROACH TO THE SPECIES CONCEPT OF STUDENTS OF NINTH GRADE OF AN EDUCATION INSTITUTION OF NEIVA CITY}

\section{Wilson Armando Guevara Calderón ${ }^{1}$}

\author{
Elías Francisco Amórtegui Cedeño²
}

\section{Resumen}

A continuación presentamos resultados preliminares del desarrollo de práctica pedagógica I al interior del Programa de Licenciatura en Educación Básica con Énfasis en Ciencias Naturales y Educación Ambiental de la Universidad Surcolombiana (Neiva-Colombia) llevada a cabo en una Institución Educativa Oficial. El grupo de trabajo estuvo conformado por estudiantes de noveno grado cuyas edades oscilan entre 13 y 16 años. Para el caso de esta ponencia nos referimos particularmente a la aproximación a las concepciones acerca del concepto especie que tienen los estudiantes. La metodología está enmarcada en una perspectiva cualitativa, empleando el análisis de contenido y haciendo uso del cuestionario. Los resultados muestran 8 tendencias, en donde Características morfológicas y Fisiológicas es la mayoritaria, implicando que los estudiantes conciben el concepto de especie a partir de las diferenciaciones relacionadas con el color, los órganos, la capacidad de vuelo y la fortaleza, entre otros; mientras que la minoritaria se refiere a Ecológica acerca de los rasgos de alimentación, hábitat, comportamiento, entre otros. Finalmente, mostramos algunas implicaciones en la enseñanza-aprendizaje de las Ciencias Naturales y proyectamos algunas posibles estrategias para favorecer al aprendizaje del concepto.

\section{Abstract}

Here are preliminary results of the development of teaching practice I into the Bachelor of Education Program with Emphasis on Basic Science and Environmental Education at the University Surcolombiana (Neiva-Colombia) held

\footnotetext{
${ }^{1}$ Estudiante de noveno semestre del Programa de Licenciatura en Educación Básica con Énfasis en Ciencias Naturales y Educación Ambiental. Universidad Surcolombiana. wilsyn826@hotmail.com

${ }^{2}$ Docente de Planta de Tiempo Completo. Programa de Licenciatura en Educación Básica con Énfasis en Ciencias Naturales y Educación Ambiental. Universidad Surcolombiana. elias.amortegui@usco.edu.co
} 
Bio-grafia Escritoy sobre la Biología y su Enseñanza.

Edición Extra-Ordinaria. ISSN 2027-1034 P.p300 - 310

Memorias del VII Encuentro Nacional de Experiencias en la Enseñanza de la

Biología y la Educación Ambiental y II Congreso Nacional de Investigación en la

Enseñanza de la Biología

in an Educational Institution Officer. The working group consisted of ninth-grade students aged between 13 and 16 years. In the case of this paper we refer particularly to the approach to the conceptions about the species concept for students. The methodology is framed in a qualitative perspective, using content analysis and using the questionnaire. The results show 8 trends, where Morphological and Physiological is the majority, implying that students conceived the concept of species from the color-related differentiations, organs, flight capacity and strength, among others, while the minority Ecological concerns about power traits, habitat, behavior, among others. Finally, we show some implications for teaching and learning of Science and project some possible strategies to promote the learning of the concept.

Palabras clave: Especie, Concepciones, Ciencias Naturales y Educación Ambiental.

Keywords: Species, Concepts, Science and Environmental Education.

\section{Introducción}

El Programa de Licenciatura en Educación Básica con Énfasis en Ciencias Naturales y Educación Ambiental, es un programa de formación inicial de docentes adscrito a la Facultad de Educación de la Universidad Surcolombiana (Neiva-Huila); éste, consta de nueve semestres y cuatro ámbitos de formación (Biología, Química, Física y Didáctica) que componen el núcleo común dentro del componente básico específico en el plan de estudios. Al interior del programa, se realizan dos prácticas pedagógicas llevadas a cabo al interior de octavo y noveno semestre respectivamente, realizándose la Práctica Pedagógica I en el nivel de la básica secundaria ${ }^{3}$ en una institución educativa de cualquier municipio del Departamento del Huila, en el área de Ciencias Naturales y Educación Ambiental; mientras que la Práctica Pedagógica II se lleva a cabo en la Educación Media, particularmente en el área de Química o Física.

Para el caso de ambas prácticas pedagógicas, tienen una duración de dieciocho semanas, de las cuales la segunda corresponde al proceso de observación del grupo de estudiantes por parte del futuro docente, en donde examina la dinámica de la clase teniendo en cuenta aspectos como los gustos, intereses, experiencias, ideas previas, dificultades de aprendizaje de los estudiantes, entre otros; las siguientes dieciséis semanas son de intervención directa junto con el

\footnotetext{
${ }^{3}$ De acuerdo a la normatividad vigente del Ministerio de Educación Nacional.
} 
Bio-grafia Escritoy sobre la Biología y su Enseñanza.

Edición Extra-Ordinaria. ISSN 2027-1034 P. p300 - 310

Memorias del VII Encuentro Nacional de Experiencias en la Enseñanza de la

Biología y la Educación Ambiental y 11 Congreso Nacional de Investigación en la Enseñanza de la Biología

estudiantado por parte del futuro docente, en donde pone en juego los conocimientos adquiridos en su formación inicial.

Para el caso de la primer semana, el practicante futuro docente realiza una previa planificación junto con el coordinador de práctica pedagógica del programa, sus compañeros y un docente asesor quien cuenta con amplia formación en investigación educativa, pedagógica y didáctica, teniendo en cuenta aspectos tales como: el modelo didáctico que orienta la práctica pedagógica, los requerimientos y requisitos de la institución educativa al igual os contenidos dentro del plan de estudios del curso a intervenir, las finalidades de la Práctica Pedagógica, las posibles estrategias a implementar de acuerdo a las bases teórico-pedagógicas que se tienen, Criterios y mecanismos de Evaluación, actividades CTS-A, uso de TICs, entre otros.

Para el caso de Colombia, las directrices planteadas por el Ministerio de Educación Nacional, contemplan dentro de los Estándares y Competencias para el grado noveno, el desarrollo de contenidos en torno a las diferentes concepciones científicas acerca del origen y evolución de los seres vivos, teniendo éste último como contenido básico el concepto de especie, su importancia, sus limitantes y en lo posible todas sus inferencias dentro de las teorías de la Evolución, es por esto que para el caso de esta ponencia nos referimos a este concepto.

En éste sentido, el concepto de Especie es fundamental para explicar, aprender, enseñar y aceptar la teoría de la evolución puesto que es una de las bases conceptuales sobre las que se ha formulado esta teoría sin mencionar que la teoría de la Evolución es fundamental en la Biología y sus implicaciones en todos los ámbitos científicos, religiosos y sociales (Jiménez, 2009), o como diría Theodosius Dobzhansky "Nada en la Biología tiene sentido si no es a la luz de la Evolución"; Así entonces, la Biología se encuentra fundamentada por la Teoría de la Evolución y esta a su vez por varios conceptos entre ellos el de especie, subrayando que en la Biología las teorías se fundamentan, se basan en conceptos.

Para el caso de esta ponencia, definimos las concepciones tal como plantea Amórtegui (2011) como sistemas de ideas que tienen los sujetos que pueden evolucionar a través de un proceso de restructuración que puede o no ser consciente, basado en la interacción con otras ideas y experiencias de los sujetos, además las concepciones originadas en un contexto particular que provocan un aumento en su grado de complejidad no se transfieren de manera automática y mecánica a otros contextos y problemas de la misma clase, pero si pueden influir en ellos, están fuertemente arraigadas en la medida que son coherentes, flexibles y funcionales y, posibilitan explicaciones causales a fenómenos físicos y al igual que las rutinas, son resistentes al cambio y consecuentemente, pueden constituir obstáculos para la transformación. Para el caso de esta ponencia cabe resaltar 
Bio-grafia Escritoy sobre la Biología y su Enseñanza.

Edición Extra-Ordinaria. ISSN 2027-1034 P. p300 - 310

Memorias del VII Encuentro Nacional de Experiencias en la Enseñanza de la

Biología y la Educación Ambiental y II Congreso Nacional de Investigación en la Enseñanza de la Biología

que nos referimos exclusivamente a las respuestas textuales de los estudiantes a partir de la aplicación de un cuestionario.

Específicamente en el campo de la enseñanza-aprendizaje de la Biología, las concepciones son fundamentales debido a que los estudiantes tienen explicaciones sobre los fenómenos biológicos que difieren de los aceptados desde el punto de vista científico, formadas de formas espontánea como consecuencia de sus percepciones sobre los fenómenos naturales (Banet, 2000).

Con relación a los antecedentes cabe destacar que por espacio no los presentamos, sin embargo para el caso del Departamento del Huila y la Ciudad de Neiva no existen no existen trabajos sobre la aproximación a las concepciones que tienen los estudiantes sobre especie ni sobres las implicaciones de estas en la enseñanza-aprendizaje de las Ciencias Naturales.

\section{Metodología}

El estudio se llevó a cabo desde un enfoque cualitativo, empleando el método de análisis de contenido en el proceso de sistematización, y usando como herramienta de recolección de información el cuestionario. El grupo objeto de estudio consistió en 29 estudiantes entre 13 y 16 años de edad, pertenecientes en su mayoría a los estratos socioeconómicos 1 y 2 que cursan noveno grado en una Institución Educativa Oficial de la ciudad de Neiva.

\section{Resultados Y Discusión}

Los hallazgos permitieron establecer 8 tendencias sobre la aproximación a las concepciones de los estudiantes. A continuación presentamos las características de cada una, así como su frecuencia y en algunos casos mostramos evidencias textuales de las respuestas del estudiantado.

ESPECIE COMO DIFERENCIA MORFOLÓGICA Y FISIOLÓGICA: Esta tendencia mayoritaria (7 estudiantes) muestra que los alumnos emplean características exclusivamente estructurales y funcionales para definir especies, tales como la capacidad del vuelo, la adquisición de fortaleza, el color, el tamaño, entre otras.

E2.C1.1.[Haciendo referencia a la diferenciación de dos especies de patos a partir de la presión de la selección natural por predación] "Pues que otros no son iguales, unos son másfuertes y pueden volar y algunos no".

Esto muestra una tendencia de agrupación poco compleja y reduccionista sobre el concepto de especie. Lo anterior puede estar relacionado con que los 
Bio-grafia Escritos sobre la Biología y su Enseñanza.

Edición Extra-Ordinaria. ISSN 2027-1034 P. p300 - 310

Memorias del VII Encuentro Nacional de Experiencias en la Enseñanza de la

Biología y la Educación Ambiental y 11 Congreso Nacional de Investigación en la

Enseñanza de la Biología

estudiantes establecen como criterios de explicación, exclusivamente aquellos relacionados con aspectos observables a nivel macroscópico y por tanto se ven en la situación de agrupar las especies de acuerdo a las características morfológicas y fisiológicas. En este sentido, los estudiantes tienen como base conceptual para la definición de especie, sus recuerdos, experiencias, lo cual está acompañado de los elementos expuestos por filósofos como Platón y Aristóteles y Naturalistas como Linneo (Jiménez, 2009), en donde dentro de la categoría especie incluyen los "individuos que comparten un cierto número de características similares". La definición de especie desde ésta tendencia, ofrece dificultades puesto que como lo diría Jiménez (2009), "Hay especies crípticas tan similares que son virtualmente indistinguibles", ofreciendo un campo de acción limitado para tal definición.

Éste concepto de Especie es normal encontrarlo en estudiantes, puesto que incluso la ciencia a través de su historia previa, ha pasado por ésta definición (Jiménez, 2009), pero brinda una idea sencilla y "palpablemente" aplicable en la vida diaria de un no científico, puesto que para un estudiante y una persona del común, le basta en su quehacer diario, agrupar las especies de acuerdo a las características morfológicas que comparten los individuos.

E10.C1.1. "Es o son grupos de animales, por lo cual cada grupo tiene sus características únicas que los dividen de otros grupos, lo que cada grupo con sus características se hacen llamar especie".

La unidad de información anterior muestra una particularidad de gran importancia dentro de las definiciones dadas por los estudiantes sobre el concepto de especie, y es el hecho de que un alto número de estudiantes encuestados, recurriendo a sus recuerdos, experiencias, preconceptos (mencionado anteriormente), ejemplifican el concepto de especie con grupos de animales, siendo los grupos de individuos de éste reino, los que mejor se "adaptan" o para los que de una forma más adecuada se acomodan a sus definiciones de especie, porque en éstos grupos -o al menos los que conocen- son "fácilmente" observables las características morfológicas y fisiológicas, desplazando los otros reinos (Plantas, Mónera, Protista y Fungí) precisamente porque son difíciles de clasificar dentro del concepto de especie que proponen, debido a que en éstos últimos abundan las "especies Crípticas tan similares que son virtualmente indistinguibles" (ejemplo las orquídeas dentro del reino de las plantas) o porque simplemente han pasado desapercibidas a través de su vida, ayudados por los profesores de ciencias naturales en las aulas de clase, que los "aburren" al momento de enseñar Botánica o en ocasiones porque ni conocen que éstos organismos existen (Ejemplo reino Mónera). 
Bio-grafía Escritos sobre la Biología y su Enseñanza.

Edición Extra-Ordinaria. ISSN 2027-1034 P. p300 - 310

Memorias del VII Encuentro Nacional de Experiencias en la Enseñanza de la

Biología y la Educación Ambiental y II Congreso Nacional de Investigación en la Enseñanza de la Biología

Para ambos casos, cabría resaltar que las posibles concepciones de los estudiantes pueden estar relacionadas con el conocimiento biológico sustantivo y sintáctico que tienen los maestros de estos estudiantes, pues autores como Amórtegui (2011), el conocimiento de la disciplina que se enseña tiene una fuerte influencia en la selección de contenidos, actividades, finalidades, entre otros, de tal forma que es posible que maestros que no cuentan con formación pos-gradual y con pocas actualizaciones en la naturaleza de la ciencias, en este caso de la Biología, aun conciban la Biología como una disciplina sin un estatus epistemológico particular y cuya estructura discursiva responde a la lógica de áreas de estudio totalmente separadas, tales como la zoología, la botánica, la fisiología, entre otros.

ESPECIE COMO DIFERENCIA REPRODUCTIVA: Aquí los estudiantes (6 alumnos) se refieren al hecho de que diferentes especies no se puedan cruzar o aparear entre ellas, sino exclusivamente con individuos de una misma especie.

E25.C1.1. "Es un grupo en el cual solo se aparean entre ellos".

E5.C1.1. "Lo que yo entiendo de especie es que cada animal no se debe aparear con otro animal que no sea de la misma especie".

Es una definición de especie más compleja que la anterior, ya que en esta no se tienen en cuenta las características morfológicas y fisiológicas observables, sino que brinda un acercamiento a una de las características fundamentales del concepto de especie trabajado por la ciencia a través de la historia, en donde se observa una definición a la que incluso Aristóteles no llegó ya que Aristóteles admitió cruzamiento entre animales de diferentes especies (Barberá, 1994)correspondiendo en parte a la definición bien aceptada de Ernst Mayr, en la que plantea que la "especie son grupos de poblaciones naturales que se reproducen en forma cruzada...". Se observa entonces, una definición cercana a la aceptada en parte por la comunidad científica, pero que no es aplicable a la totalidad de seres vivos, puesto que no aplica para organismos con reproducción asexual (Mayr, 2006).

ESPECIE COMO DIFERENCIA DE REPRODUCCIÓN Y HERENCIA: Aquí los estudiantes (6 alumnos) tienen como criterio para la definición del concepto especie el apareamiento entre organismos de la misma especie y además el hecho de generar una descendencia fértil y la capacidad de heredar características.

E12.C1.1."Conjunto de organismos que se pueden cruzar/reproducirse para heredar características a las otras generaciones". 
Bio-grafia Escritos sobre la Biología y su Enseñanza.

Edición Extra-Ordinaria. ISSN 2027-1034 P. p300 - 310

Memorias del VII Encuentro Nacional de Experiencias en la Enseñanza de la

Biología y la Educación Ambiental y 11 Congreso Nacional de Investigación en la

Enseñanza de la Biología

Los estudiantes que se ubican en ésta tendencia, abarcan un punto adicional en comparación con la agrupación anterior y es que, además del cruce sólo entre individuos de la misma especie, enmarcan el hecho de que pueden tener descendencia, y particularmente una descendencia fértil con el fin de que se hereden características de generación en generación; lo anterior corresponde a una definición cercada a los planteamientos de Mayr (2006), quién afirma que las especies "... son grupos de poblaciones naturales que se reproducen en forma cruzada..." y a Barberá (1994) quién afirma: "En el pensamiento de Aristóteles lo que realmente tienen en común todos los individuos que constituyen una especie, además del término que los describe, es la posibilidad de procrear individuos semejantes a ellos"; sin embargo, lo anterior explica tan solo la reproducción sexual. Lo anterior corresponde a la definición de especie como concepto biológico en el cual, los individuos que en caso de encontrarse en la naturaleza podrían producir descendencia fértil constituyen una especia (Galli, 2010).

ESPECIE COMO DIFERENCIA FILOGENÉTICA: Aquí los estudiantes (3 alumnos) agrupan a los individuos de la misma especie de acuerdo a la presencia de ancestros comunes.

\section{E9.C1.1."La especie es si proviene o no de un ancestro en común".}

Éstas respuestas presentan similitudes con las definiciones dadas por diferentes autores como Jiménez (2009), cuando intentan dar solución a una de las limitaciones del concepto enmarcado en la reproducción y la herencia, tales como la aplicación del concepto cuando se trata de especies fósiles, en donde los autores como Simpson (1961) citado por Jiménez (2009) dice: "Una especie evolutiva es un linaje que evoluciona en forma separada de otras y con su propio papel y tendencias evolutivos unitarios", pero "¿cómo saber cuál es su papel evolutivo (en el futuro) o sus tendencias históricas?"; además el concepto expuesto por los estudiantes aquí encuestados sólo se quedan en parte del concepto expuesto por Simpson, lo que lo lleva a ser menos válido, puesto que miles de Especies pueden evolucionar de un ancestro en común. Sin embargo, van más allá y ofrecen una de las características de las especies visto desde la Evolución y es precisamente el hecho de que las especies pueden cambiar y variar a través del tiempo y originar especies diferentes a partir de procesos de especiación.

ESPECIE COMO DIFERENCIA GENÉTICA: Aquí los estudiantes (2 alumnos) se refierena que diferencias entre el material genético, permite distinguir entre una $u$ otra especie.

E1.C1.1."Es un tipo especificado de animal, organismo, etc. los cuales se diferencian genéticamente por el ADN". 
Bio-grafía Escritos sobre la Biología y su Enseñanza.

Edición Extra-Ordinaria. ISSN 2027-1034 P. p300 - 310

Memorias del VII Encuentro Nacional de Experiencias en la Enseñanza de la

Biología y la Educación Ambiental y 11 Congreso Nacional de Investigación en la

Enseñanza de la Biología

Lo anterior corresponde a los planteamientos de Mayr (2006), quién afirma: "Especies son grupos de poblaciones naturales que se reproducen en forma cruzada y que se hallan reproductivamente (Genéticamente) aisladas de otros grupos semejantes"; sin embargo, el problema con la definición de los estudiantes, es que si sólo se busca la diferenciación de las especies teniendo en cuenta su material genético, no podría explicar el hecho de que existen diferencias en el ADN incluso en individuos y/o poblaciones de la misma especie. A pesar de esto, es favorable que los alumnos expongan en parte una de las características utilizadas para diferenciar las especies (Jiménez, 2009).

ESPECIE COMO DIFERENCIACIÓN REPRODUCTIVA Y MORFOLÓGICA: Aquí los estudiantes (2 alumnos) hacen alusión tanto a elementos a nivel micro como el fenómeno de la reproducción y a nivel macro como las características morfológicas.

E14.C1.1."Conjunto de individuos que se aparean entre sí y con características similares".

Como ya se ha mencionado anteriormente, definir el concepto sólo desde el punto de vista de la Reproducción o sólo desde la Morfología de los individuos que forman una especie origina limitaciones tanto en el Conocimiento Biológico como en su enseñanza y aprendizaje, pero generar el concepto desde la unión de las dos características amplía las aplicaciones del concepto, puesto que, desde la Morfología, la limitación fundamental es el hecho de que "Hay especies Crípticas tan similares que son virtualmente indistinguibles" (Jiménez, 2009), pero si a ésta característica se adiciona la reproducción sólo entre individuos de la misma especie, soluciona la limitación a las características Morfológicas, sin embargo queda la limitante de la reproducción y es el hecho de que sólo aplica a especies con reproducción sexual.

ESPECIE COMO DIFERENCIACIÓN GENÉTICA Y MORFOLÓGICA: Aquí loS estudiantes (2 alumnos) consideran que los individuos de una misma especie son genéticamente iguales y además comparten características morfológicas.

\section{E26.C1.1."Es una forma de separar los animales por sus genes, ADN o} características y poderlo tener en un grupo seleccionado con demás animales parecidos al de su misma especie".

Al igual que la anterior, esta tendencia ofrece dos características fundamentales para clasificar las especies y es el hecho de diferenciarlos genética y morfológicamente; Sin embargo son afirmaciones que presentan al menos dos limitantes sustanciales para definir o distinguir una especie de otra(s) y son, por un lado el hecho de que existen diferencias genéticas incluso en los individuos de la 
Bio-grafía Escritos sobre la Biología y su Enseñanza.

Edición Extra-Ordinaria. ISSN 2027-1034 P. p 300 - 310

Memorias del VII Encuentro Nacional de Experiencias en la Enseñanza de la

Biología y la Educación Ambiental y II Congreso Nacional de Investigación en la Enseñanza de la Biología

misma especie y el segundo es que "Hay especies Crípticas tan similares que son virtualmente indistinguibles" (Jiménez, 2009).

ESPECIE COMO DIFERENCIACIÓN ECOLÓGICA: Aquí tan solo un estudiante argumenta que los individuos de una especie comparten aspectos ecológicos tales como el hábitat, sus relaciones comportamentales, alimentación, entre otros.

E15.C1.1."Conjunto de seres vivos (reino animal) los cuales comparten las mismas características, rasgos, actitudes, hábitat, comportamiento y alimento".

Por último, esta tendencia ofrece una explicación conceptual de especie desde un punto de vista que a nuestro parece reúne algunos aspectos ecológicos en donde se tienen en cuenta, además de las características Morfológicas, características que consideramos son propias de la Ecología tales como el nicho, las interacciones entre los individuos y el medio, las condiciones y los recursos, entre otras; sin embargo, la problemática de ésta definición radica en que los ambientes y por ende sus condiciones y recursos son tomados en la mayoría de los casos por más de una especie, presentando así interacciones entre poblaciones de especies diferentes.

\section{Posibles Estrategias De Enseñanza-Aprendizaje}

Por una parte, es fundamental que los maestros tengan un conocimiento lo suficientemente complejo, argumentado y actualizado sobre el concepto especie de tal forma que su enseñanza no se reduzca a la explicación de dicho concepto exclusivamente desde una de las tendencias que hemos mostrado en este trabajo, sino que presente a sus estudiantes toda una gama de criterios de explicación (morfológico, genético, reproductivo y ecológico, entre otros) sobre este concepto.

De acuerdo a Jiménez (2003) y es fundamental tratar en la clase de ciencias las explicaciones biológicas desde la perspectiva de las narrativas históricas, que si bien no pueden probar los fenómenos evolutivos, pueden considerarse como elementos de discusión y debate entre los estudiantes.

Por último, podrían emplearse situaciones problema en la que los estudiantes tuvieran que dar cuenta de tales como las planteadas por Galli (2010): generación de osos pardos y osos blancos en la región ártica, generación de peces en lagos sin luz, generación de felinos sociales, entre otros.

\section{Conclusiones}


Bio-grafía Escritos sobre la Biologia y su Enseñanza.

Edición Extra-Ordinaria. ISSN 2027-1034 P.p300 - 310

Memorias del VII Encuentro Nacional de Experiencias en la Enseñanza de la

Biología y la Educación Ambiental y II Congreso Nacional de Investigación en la

Enseñanza de la Biología

Con relación a las concepciones de los estudiantes sobre el concepto de Especie, se observa que presentan diferentes tendencias, incluidas todas ellas dentro de las concepciones científicamente aceptadas para el concepto de Especie, lo que lleva a pensar, que su formación académica en cuanto al concepto de Especie se refiere, es en general buena. Sin embargo las concepciones que tienen no son ni única ni completa, entendiéndose esto, porque al igual que en la concepción Universal del Concepto de especie en la Biología, no existe unanimidad sobre el concepto, Se debe entonces, trabajar e implementar toda la "gama" de concepciones que se tienen como válidas por la comunidad científica, en el aula de clases --teniendo presente que el concepto de Especie es uno de los pilares de la Teoría de la Evolución para que de ésta forma el estudiante tenga una gama de posibilidades para explicar los fenómenos de especiación y por lo tanto no se debe cerrar el concepto de especie a uno sólo y único, puesto que aún no se ha "creado" un concepto que defina en todos los contextos lo que significa una especie, sino una "gama" de Conceptos.

Lo anterior muestra la importancia de favorecer procesos formativos en los cuales los estudiantes pongan en evidencia sus concepciones, pero además a través de discusiones puedan detectarlas, modificarlas y re plantearlas en aras de una enseñanza-aprendizaje mucho más compleja y enriquecida desde perspectivas constructivistas.

\section{Bibliografía}

- Amórtegui, E (2011). Concepciones sobre prácticas de campo y su relación con el conocimiento profesional del profesor, de futuros docentes de Biología de la Universidad Pedagógica Nacional. Tesis para optar al título de Magíster en Educación. Bogotá DC: Universidad Pedagógica Nacional.

- Banet, E (2000). Enseñanza aprendizaje del conocimiento Biológico. En: Perales, F \& Cañal, P (2000). Didáctica de las ciencias experimentales. Madrid: Acoy.

- Barberá, O (1994). Historia del concepto de especie en Biología. Enseñanza de las Ciencias, 12 (3), 417-430.

- Gallí, L (2010). La teoría de la evolución. En: Meinardi, E; Gallí, L; Revel, A; Plaza, M (2010). Educar en ciencias. Buenos Aires: Paidos.

- Jiménez, M (2003). La enseñanza y el aprendizaje de la Biología. En: Jiménez, $\mathrm{M}$ et al (2003). Enseñar Ciencias. Barcelona: Graó. 
Bio-grafía Escritos sobre la Biología y su Enseñanza.

Edición Extra-Ordinaria. ISSN 2027-1034 P.p 300 - 310

Memorias del VII Encuentro Nacional de Experiencias en la Enseñanza de la

Biología y la Educación Ambiental y 11 Congreso Nacional de Investigación en la Enseñanza de la Biología

- Jiménez, M (2009). Los conceptos de población y Especies en la enseñanza de la Biología: concepciones, dificultades y perspectivas. Tesis para optar al título de Doctor en Didáctica de las Ciencias Experimentales: Granada: Universidad de Granada.

- Mayr, E (2006).Por qué es única la Biología: Consideraciones sobre la autonomía de una disciplina científica. Buenos Aires: Katz Editores. 